\title{
Correlação entre a gravidade da doença degenerativa lombar e o alinhamento espinopélvico
}

\section{Correlation between the Severity of the Lumbar Degenerative Disease and Sagittal Spinopelvic Alignment}

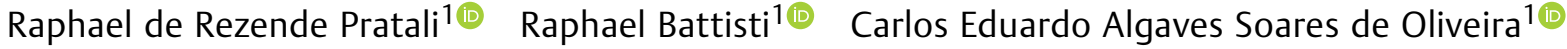 \\ Daniel Augusto Carvalho Maranho ${ }^{2(1)}$ Carlos Fernando P. S. Herrero ${ }^{20}$
}

\footnotetext{
1 Serviço de Ortopedia e Traumatologia, Hospital do Servidor Público Estadual de São Paulo, São Paulo, SP, Brasil

2 Departamento de Biomecânica, Medicina e Reabilitação do Aparelho Locomotor, Hospital das Clínicas de Ribeirão Preto, Ribeirão Preto, SP, Brasil
}

Rev Bras Ortop 2022;57(1):41-46.
Endereço para correspondência Raphael de Rezende Pratali, Rua Pedro de Toledo 1.800, Vila Clementino, 04039-901, São Paulo, SP, Brasil (e-mail: pratalir@gmail.com).

\section{Resumo \\ Palavras-chave \\ - espondilose \\ - coluna vertebral \\ - dor lombar \\ - classificação \\ - radiografia \\ Objetivo Avaliar o impacto da graduação da doença degenerativa lombar (DDL) sobre o alinhamento sagital espinopélvico. \\ Métodos Ao todo, 130 pacientes (dade média: 57 anos; 75\% do sexo feminino) com dor lombar associada a DDL foram prospectivamente incluídos. A gravidade da DDL foi definida pelos seguintes achados nas radiografias anteroposterior e de perfil da coluna lombar: osteofitose; perda de altura do disco intervertebral; esclerose na placa vertebral terminal; número de segmentos afetados; deformidades; e instabilidade objetiva. Os pacientes foram graduados segundo a DDL da seguinte maneira: grau 0 -ausência de sinais de DDL na coluna lombar; grau I - sinais de DDL em até dois segmentos; grau II - envolvimento em três ou mais segmentos; grau III - quando associada a escoliose, espondilolistese ou laterolistese. Parâmetros radiográficos espinopélvicos, como incidência pélvica (IP), lordose lombar (LL), discrepância entre a IP e a LL (IP-LL), versão pélvica (VP), e eixo vertical sagital (EVS) foram analisados de acordo com os graus de DDL. \\ Resultados Houve diferença nos parâmetros radiográficos comparando-se os graus de DDL, com os pacientes de grau III apresentando maiores valores de EVS $(p=0,001)$ e $\operatorname{VP}(p=0,0005)$, o que denota maior inclinação anterior do tronco e maior retroversão pélvica do que os pacientes de graus 0 e I. Pacientes de grau III também apresentaram maiores valores de IP-LL, o que denota perda da lordose relativa ao valor da IP, do que pacientes grau I $(p=0,04)$. \\ Conclusão Pacientes com DDL mais grave demonstraram uma tendência a maior desalinhamento sagital espinopélvico comparados com pacientes com graus mais leves.}

recebido

22 de Julho de 2020

aceito

03 de Novembro de 2020
DOI https://doi.org/

10.1055/s-0041-1729579. ISSN 0102-3616. (c) 2022. Sociedade Brasileira de Ortopedia e Traumatologia. All rights reserved.

This is an open access article published by Thieme under the terms of the Creative Commons Attribution-NonDerivative-NonCommercial-License, permitting copying and reproduction so long as the original work is given appropriate credit. Contents may not be used for commercial purposes, or adapted, remixed, transformed or built upon. (https://creativecommons.org/ licenses/by-nc-nd/4.0/)

Thieme Revinter Publicações Ltda., Rua do Matoso 170, Rio de Janeiro, RJ, CEP 20270-135, Brazil 


\begin{abstract}
Keywords

- spondylosis

- spine

- lumbar pain

- classification

- radiography

Objective To evaluate the impact of the severity of lumbar degenerative disease (LDD) on sagittal spinopelvic alignment.

Methods In total, 130 patients (mean age: 57 years; $75 \%$ female) with LDD-associated low-back pain were prospectively included. The severity of the LDD was defined by the following findings on anteroposterior and lateral lumbar spine radiographs: osteophytosis; loss of of height of the intervertebral disc; terminal vertebral plate sclerosis; number of affected segments; deformities; and objective instability. The disease was classified as follows: grade 0 -absence of signs of LDD in the lumbar spine; grade I - signs of LDD in up to two segments; grade II - three or more segments involved; grade III - association with scoliosis, spondylolisthesis, or laterolisthesis. Spinopelvic radiographic parameters, including pelvic incidence (PI), lumbar lordosis (LL), discrepancy between the PI and LL (PI-LL), pelvic tilt (PT), and sagittal vertical axis (SVA), were analyzed according to the LDD grades.

Results The radiographic parameters differed according to the LDD grades; grade-III patients presented higher SVA $(p=0.001)$ and PT $(p=0.0005)$ values, denoting greater anterior inclination of the trunk and pelvic retroversion when compared to grade- 0 andgrade-I subjects. In addition, grade-III patients had higher PI-LL values, which indicates loss of PI-related lordosis, than grade-I subjects $(p=0.04)$.

Conclusion Patients with more severe LDD tend to present greater spinopelvic sagittal misalignment compared to patients with a milder disease.
\end{abstract}

\section{Introdução}

A análise dos parâmetros radiográficos do alinhamento sagital espinopélvico se tornou imprescindível na avaliação e no tratamento da deformidade da coluna vertebral no adulto (DCVA). ${ }^{1}$ A importância desses parâmetros radiográficos provém da forte correlação demonstrada com indicadores de qualidade de vida e capacidade funcional, ${ }^{2-4}$ que foi a base para o desenvolvimento do sistema de classificação mais utilizado atualmente para DCVA, conhecido como Scoliosis Research Society-Schwab (SRS-Schwab). ${ }^{5}$ Mais recentemente, a relevância clínica do SRS-Schwab foi demonstrada, sendo que a intensidade da limitação funcional e a escolha do tipo de tratamento são influenciadas pelos subtipos do SRS-Schwab e seus modificadores. ${ }^{6}$

A frequência da DCVA está relacionada com a idade populacional, sendo maior a prevalência em indivíduos idosos. ${ }^{7,8}$ Sendo assim, como esperado na população idosa, outras condições podem estar presentes concomitantemente à DCVA, potencialmente confundindo a limitação funcional desses indivíduos. Entre tais condições, podemos destacar a doença degenerativa lombar (DDL), cuja fisiopatologia envolve alterações anatômicas e estruturais nos discos intervertebrais, nas articulações facetárias, e nos corpos vertebrais. ${ }^{9}$ Tais alterações degenerativas podem associar-se com deformidades da coluna vertebral, instabilidades como espondilolistese e estreitamento do canal ou foraminal, e podem ainda levar à compressão de elementos neurais, resultando em importante dor lombar axial e/ou dor radicular. ${ }^{10}$

Nesse contexto, faltam estudos que avaliem a interferência da DDL nas alterações dos parâmetros radiográficos espino- pélvicos, assim como com a correlação desses parâmetros com a ocorrência de sintomas e a limitação funcional. O objetivo do presente estudo foi avaliar a relação entre a gravidade da DDLe o alinhamento sagital espinopélvico.

\section{Materiais e Métodos}

\section{População}

Trata-se de um estudo transversal que incluiu uma coorte de pacientes recrutados prospectivamente, de forma não consecutiva, em atendimento ambulatorial. O estudo foi aprovado pelo Comitê de Ética em Pesquisa do serviço onde foi conduzido (CAAE: 18013219.7.0000.5463), e os pacientes assinaram um termo de consentimento livre esclarecido. Foram incluídos pacientes adultos (com idade $\geq 18$ anos) com queixa de dor lombar com ou sem sintomas de radiculopatia, e que apresentavam sinais radiográficos de DDL. Os critérios de exclusão foram: cirurgia prévia na coluna vertebral ou sistema nervoso central; doenças neurológicas ou neuromusculares; histórico de trauma ou doença neoplásica na coluna vertebral; queixa de incapacidade no quadril/joelho/tornozelo/pé que pudesse alterar as posições articulares; e radiografia inadequada que não permitisse a visualização da segunda vértebra cervical (C2) à cabeça do fêmur, ou sem resolução suficiente para análise de sinais radiográficos de DDL.

\section{Graduação da Doença Degenerativa Lombar}

Foram consideradas imagens nas incidências de frente e de perfil do segmento lombar da coluna vertebral, extraídas de exames de radiografia total da coluna vertebral (panorâmica). Somente foram consideradas imagens digitalizadas, no formato 
digital imaging and communications in medicine (DICOM, comunicação de imagens digitais em medicina), que permitissem ampliação mantendo a qualidade e resolução, com maior precisão para avaliação nível a nível de todo o segmento lombar. Todos os exames foram realizados no mesmo serviço de radiologia e seguindo técnica padronizada: postura confortável em ortostatismo, com os ombros em elevação de $45^{\circ}$ e cotovelos flexionados, repousando as pontas dos dedos sobre as clavículas ou sobre a face. ${ }^{11}$

A gravidade da DDL foi definida por meio da escala de graduação recentemente publicada, que considera o exame de radiografia total da coluna. ${ }^{12}$ Tal escala determina a gravidade da DDL com base na análise da presença ou ausência dos principais sinais radiográficos de DDL, incluindo osteofitose marginal no corpo vertebral, perda da altura do disco intervertebral, esclerose e cistos subcondrais nas placas terminais, assim como o número de segmentos vertebrais acometidos. ${ }^{12}$ Também foram considerados a ausência ou presença de escoliose ou sinais de instabilidade objetiva, incluindo espondilolistese e laterolistese. Cada radiografia foi avaliada por dois examinadores independentes, ambos ortopedistas estagiários em cirurgia de coluna vertebral (com um e dois anos de experiência), e, de acordo com esses critérios, os pacientes foram graduados da seguinte maneira (-Figura 1):

- Grau 0: ausência de sinais de DDL.

- Grau I: presença de sinais de DDL em um ou dois segmentos, sem escoliose ou sinais de instabilidade.

- Grau II: presença de sinais de DDL em três ou mais segmentos, sem escoliose ou sinais de instabilidade.

- Grau III: presença de sinais de DDL associados com escoliose (inclinação coronal $\geq 30^{\circ}$, medida pela técnica de Cobb) e/ou sinais de instabilidade, como laterolistese ( $>2 \mathrm{~mm}$ ) e espondilolistese (pelo menos grau 2 ).
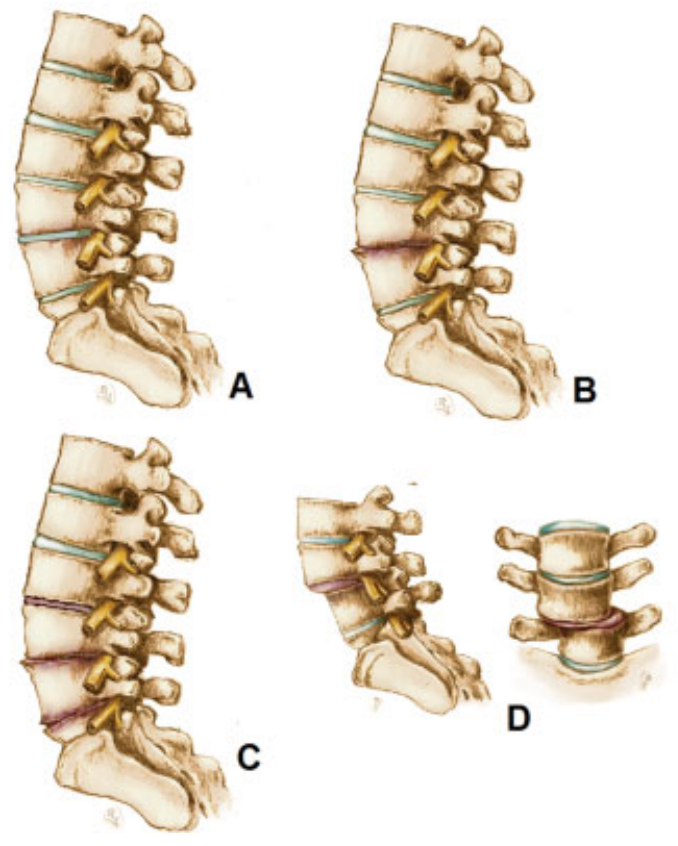

Fig. 1 llustração da escala de graduação radiográfica da doença degenerativa lombar (DDL). (A) Grau zero; (B) grau I; (C) grau II; (D) grau III.
Avaliação dos Parâmetros Radiográficos Espinopélvicos

Os parâmetros radiográficos foram medidos utilizando-se o software Surgimap Spine (Nemaris, Inc., Nova York, NY, US), ferramenta validada para avaliação radiográfica da coluna vertebral. ${ }^{13}$ Os parâmetros considerados foram: o eixo vertical sagital (EVS), calculado como a distância entre a linha de prumo de C7 e o canto posterossuperior do sacro; a versão pélvica (VP), o ângulo entre a linha que tangencia o platô superior do sacro e o plano horizontal; a incidência pélvica(IP), o ângulo entre uma linha perpendicular ao centro do platô superior do sacro e uma linha do centro do platô superior do sacro e o centro das cabeças do fêmur; a lordose lombar (LL), o ângulo de Cobb sagital entre o platô superior de L1 e o platô superior de S1; e a discrepância entre IP e LL (IP-LL). ${ }^{1}$ Os parâmetros foram medidos por dois examinadores independentes (os mesmos que graduaram a DDL), e, havendo eventuais discrepâncias entre as duas medidas, uma terceira medida foi realizada. $\mathrm{O}$ valor de cada parâmetro foi obtido pela média das medidas dos dois examinadores.

\section{Análise Estatística}

A análise estatística foi realizada utilizando-se o programa $R(R$ Foundation for Statistical Computing, Viena, Áustria), versão 3.4.9. Após a graduação dos pacientes quanto à DDL, os parâmetros radiográficos espinopélvicos foram comparados entre os 4 graus, por meio do cálculo da análise de variância (analysis of variance, ANOVA, em inglês) com pós-teste de Bonferroni. Foi considerado nível de significância de $5 \%$, com valores de $p<0,05$ sendo estatisticamente significativos.

\section{Resultados}

\section{População, Dados Demográficos e Graduação da DDL}

De um total de 138 exames radiográficos, foram considerados 130 pacientes que preencheram os critérios de inclusão e tinham exame com suficiente resolução de imagem. No total, havia 97 (75\%) mulheres, e a média de idade da amostra era de 57 anos (desvio padrão [DP]: 14,6 anos), variando entre 18 e 95 anos. Com relação à escala de graduação da DDL, 12 (9\%) pacientes eram grau 0, 41 (32\%) eram grau I, 43 (33\%) eram grau II, e 34 (26\%) eram grau III. Houve diferença significante da idade entre os graus de $\operatorname{DDL}(p<0,001)$ ( - Figura 2), sendo que os pacientes do grau I (média: 51 anos; DP: 10 anos)

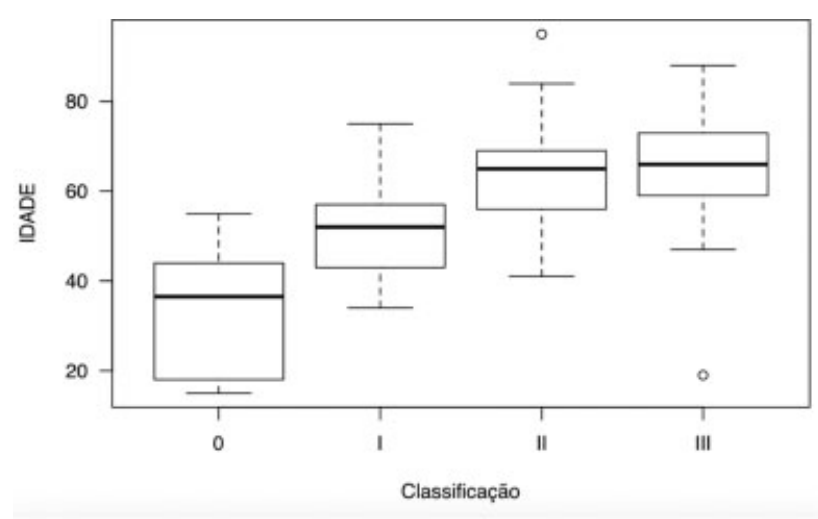

Fig. 2 Comparação da idade entre os graus de DDL. 


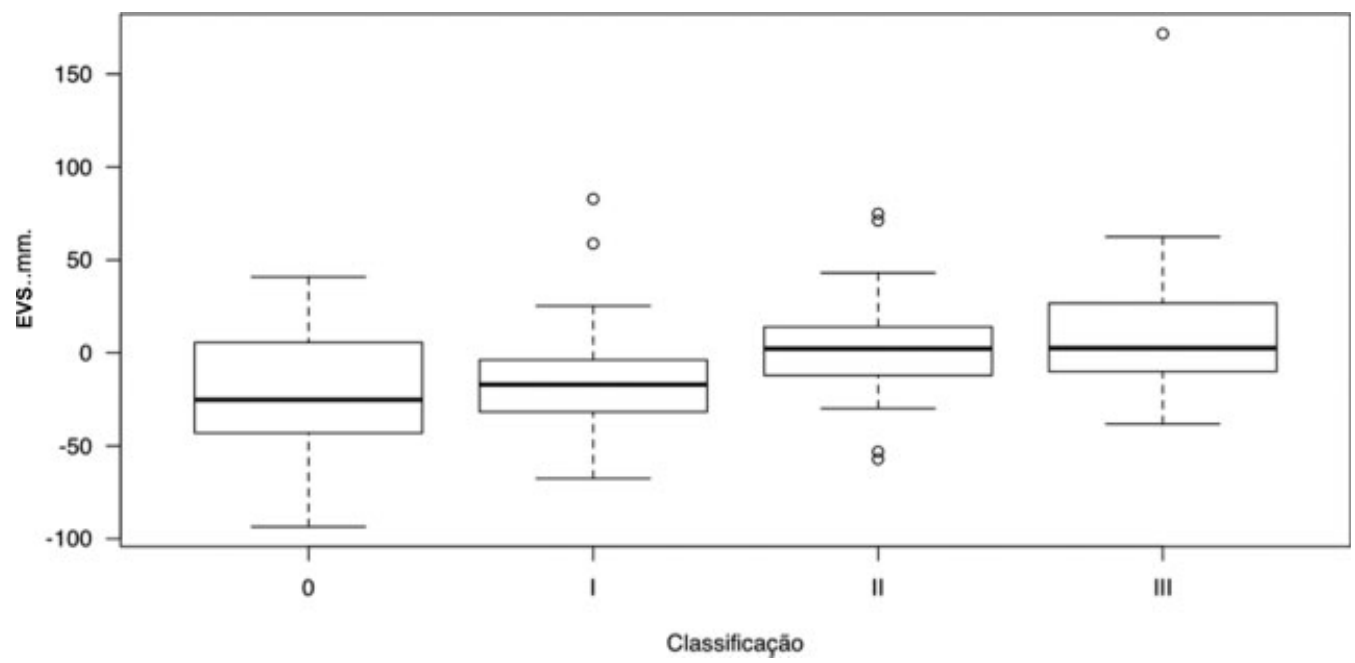

Fig. 3 Comparação do eixo vertical sagital (EVS) entre os graus de DDL.

eram mais velhos do que os do grau 0 (média: 33 anos; DP: 13,6 anos) $(p<0,001$ ); os do grau II (média: 63 anos; DP: 10,6 anos), mais velhos do que os do grau I $(p<0,001)$; e sem diferença significante entre os graus II e III (média: 65 anos; DP: 12,2 anos) $(p=1)$.

\section{Parâmetros Radiográficos Espinopélvicos}

Houve diferença no EVS entre os graus de DDL (- Figura 3), sendo que pacientes classificados como grau III apresentaram média maior, ou seja, maior inclinação anterior do tronco, do que os de grau I $(p=0,008)$ e grau $0(p=0,03)$ (-Tabela 1 ). Também houve diferença na VP entre os graus de DDL (-Figura 4), e pacientes classificados como grau III tiveram média maior, maior retroversão pélvica, do que aqueles de grau I $(p<0,001)$ e grau $0(p=0,01)(-$ Tabela 1$)$. Comparando-se os valores da IP-LL, houve diferença entre os graus de DDL (-Figura 5), sendo que a média no grau III foi maior do que no grau I $(p=0,04)$. Não houve diferença significante entre os graus de DDL comparando-se os valores da IP e da LL (-Tabela 1).

\section{Discussão}

Atualmente, o mundo enfrenta uma mudança demográfica global, com o envelhecimento da população relacionado com o aumento da expectativa de vida, e, assim, aumento dos problemas de saúde relacionados à idade. ${ }^{7,8}$ Muitas vezes, pode haver sobreposição de mais de um problema, como frequentemente ocorre na coluna vertebral, em que a DDL pode se associar com a perda do alinhamento sagital. ${ }^{14}$ Diversos artigos $^{2-4,6,10,14}$ sugerem associação tanto da DDL quanto da perda do alinhamento sagital com sintomas de dor e incapacidade funcional, mas faltam estudos demonstrando a real relação entre a gravidade da DDL e a perda do alinhamento sagital, assim como qual o papel de cada componente na etiologia dos sintomas dos pacientes.

Recentemente, foi apresentado um sistema de graduação da DDL por meio da análise de achados radiográficos dos segmentos lombares vistos em radiografias totais da coluna (panorâmicas). ${ }^{12}$ A motivação para o desenvolvimento dessa escala de graduação foi justamente poder avaliar no mesmo exame, a radiografia total da coluna, tanto a DDL quanto os parâmetros do alinhamento sagital espinopélvico. ${ }^{12}$ $\mathrm{O}$ advento da análise digital das imagens radiográficas permite ampliação suficiente, mantendo a resolução, para se avaliar os sinais de doença degenerativa nos segmentos lombares nesses exames.

Quanto à DCVA, o sistema de classificação mais utilizado é o SRS-Schwab, que descreve o padrão da deformidade no plano coronal, e também considera três parâmetros radiográficos

Tabela 1 Valores dos parâmetros radiográficos espinopélvicos no total da amostra e nos graus da escala da doença degenerativa lombar

\begin{tabular}{|c|c|c|c|c|c|c|}
\hline & $\begin{array}{l}\text { TOTAL } \\
\text { M (DP) }\end{array}$ & $\begin{array}{l}\text { GRAU } 0 \\
\text { M (DP) }\end{array}$ & $\begin{array}{l}\text { GRAU I } \\
\text { M (DP) }\end{array}$ & $\begin{array}{l}\text { GRAU II } \\
\text { M (DP) }\end{array}$ & $\begin{array}{l}\text { GRAU III } \\
\text { M (DP) }\end{array}$ & $p$ \\
\hline EVS (mm) & $-3,4(32,8)$ & $-21,1(36,6)$ & $-14,9(27,7)$ & $2,6(26)$ & $8,9(38,4)$ & 0,001 \\
\hline $\operatorname{VP}\left({ }^{\circ}\right)$ & $17(11,4)$ & $11,9(9,6)$ & $13,3(10,4)$ & $17,1(9,4)$ & $23,3(12,8)$ & $<0,001$ \\
\hline $\operatorname{IP}\left({ }^{\circ}\right)$ & $53,5(14,8)$ & $49,3(19,3)$ & $51,4(16)$ & $53,2(12)$ & $57,8(14,3)$ & 0,191 \\
\hline $\operatorname{LL}\left({ }^{\circ}\right)$ & $56,7(13,3)$ & $58,1(10,5)$ & $57,7(13,5)$ & $56,4(11,7)$ & $55,5(15,8)$ & 0,884 \\
\hline IP-LL $\left({ }^{\circ}\right)$ & $-3,4(14,8)$ & $-8,7(10,2)$ & $-6,8(13)$ & $-3,3(13)$ & $2,3(18,5)$ & 0,03 \\
\hline
\end{tabular}

Abreviaturas: DP, desvio padrão; EVS, eixo vertical sagital; IP, incidência pélvica; IP-LL, discrepância entre IP e LL; LL, lordose lombar; M, média; VP, versão pélvica. 


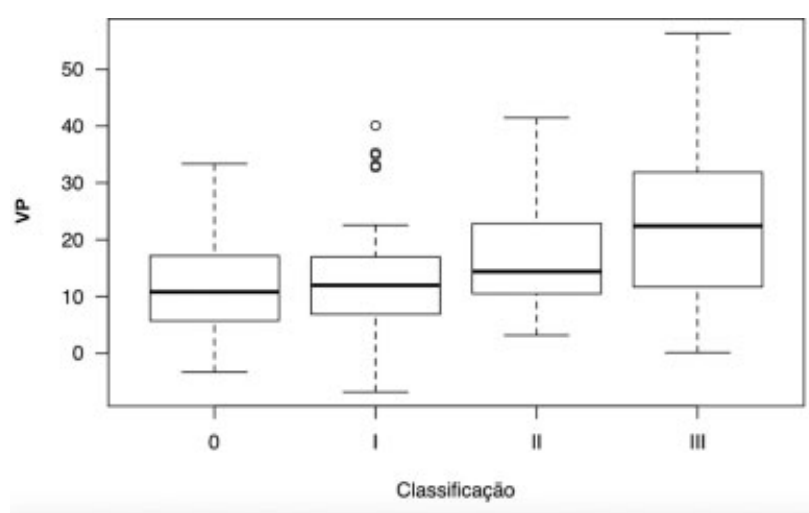

Fig. 4 Comparação da versão pélvica (VP) entre os graus de DDL.

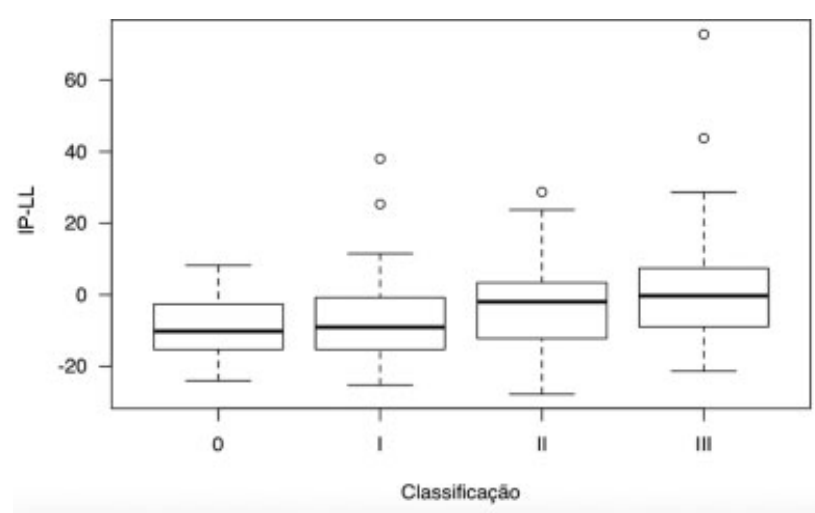

Fig. 5 Comparação da discrepância entre a incidência pélvica e a lordose lombar (IP-LL) entre os graus de DDL.

do alinhamento espinopélvico como modificadores sagitais. ${ }^{5}$ Esses modificadores incluem os parâmetros EVS, VP e IP-LL, que são classificados de acordo com a gravidade da perda do alinhamento sagital. Terran et al. ${ }^{6}$ observaram que havia forte correlação entre a piora desses modificadores, o que significa piora no alinhamento sagital espinopélvico, e piores indicadores de qualidade de vida, assim como com a decisão pelo tratamento cirúrgico e o emprego de cirurgias mais complexas.

Os resultados do presente estudo mostraram aumento nos valores justamente dos parâmetros EVS, VP e IP-LL, que correspondem aos modificadores sagitais da classificação SRS-Schwab, nos pacientes com DDL classificados como grau III segundo o esquema de graduação considerado. $O$ aumento desses parâmetros sugere piora do alinhamento sagital espinopélvico, com maior inclinação anterior do tronco (EVS) e maior retroversão da pelve (VP) como possível mecanismo de compensação à inclinação anterior. Além disso, a relação entre a LL e a IP está bem estabelecida, de modo que o valor da LL de cada indivíduo deve ser harmônico com o valor da IP;4,5 daí a importância do parâmetro IP-LL. Valores maiores da discrepância IP-LL significam perda da LL em relação ao que se espera a partir da IP., ${ }^{4,5}$ No presente estudo, pode-se perceber que os pacientes de grau III têm uma tendência à perda do alinhamento sagital espinopélvico, com aumento do valor do EVS, o que denota inclinação anterior do tronco, e aumento da VP, com retroversão da pelve como possível mecanismo compensatório. Além disso, houve aumento da IP-LL, o que significa falta relativa de LL em relação ao valor da IP.

Pacientes classificados como grau III eram também mais velhos do que os pacientes de graus $0 \mathrm{e} \mathrm{I}$, o que pode ter influenciado o aumento dos valores do EVS, da VP e de IP-LL. Um estudo recente ${ }^{15}$ demonstrou que indivíduos assintomáticos apresentam aumento do EVS e da VP com o aumento da idade. Em outro estudo, Lafage et al. ${ }^{16}$ observaram que, além de o alinhamento sagital se alterar conforme a idade, isso não necessariamente se relaciona com o aparecimento de sintomas e incapacidade funcional. Frente a esses dados, devemos questionar se os limiares dos parâmetros radiográficos considerados como modificadores sagitais do sistema de classificação SRS-Schwab deveriam ser ajustados com relação à idade. $^{16}$

Até o momento, desconhece-se se haveria influência dos achados da DDL na correlação entre a perda do alinhamento sagital e a ocorrência de dor e incapacidade funcional, como ficou demonstrado haver influência da idade na correlação entre alinhamento sagital e dor/incapacidade funcional. Alguns achados da DDL, principalmente enquadrados no grau III da escala de graduação empregada no presente estudo, que incluem escoliose e instabilidade objetiva (espondilolistese e laterolistese), poderiam confundir tal correlação, pelo fato de serem fatores predisponentes, por si só, à alteração dos parâmetros de alinhamento espinopélvico. Isso pode gerar um fator de confusão quanto a o que tem maior relevância na perda do alinhamento sagital, se $o$ avanço da DDL ou a presença da deformidade, e precisa ser mais bem estudado.

Podemos, no entanto, identificar limitações no presente estudo, sobretudo por se tratar de uma análise observacional exclusivamente radiográfica, sem considerar aspectos clínicos, como indicadores de qualidade de vida, nessa correlação entre os parâmetros radiográficos do alinhamento espinopélvico e a graduação da DDL. Podemos identificar também um viés de seleção na inclusão dos participantes, todos provenientes de um ambulatório de ortopedia, apresentando alguma queixa relacionada com a coluna vertebral, em vez de indivíduos assintomáticos estratificados pela idade.

\section{Conclusão}

O estudo da correlação entre o grau da DDL e os parâmetros radiográficos do alinhamento espinopélvico mostrou que pacientes com DDL mais grave têm uma tendência à perda do alinhamento sagital, com maior inclinação anterior do tronco e retroversão da pelve como possível mecanismo compensatório, além de hipolordose lombar, relativa ao valor da IP. O esquema de graduação da DDL utilizado pode servir como base para futuros estudos, com o objetivo de compreender melhor o papel da DDL no alinhamento sagital em indivíduos assintomáticos e estratificados por idade, assim como independente das deformidades incluídas no grau III.

Suporte Financeiro

O presente estudo não recebeu qualquer tipo de financiamento. 
Conflito de Interesses

Os autores declaram não haver conflito de interesses.

\section{Referências}

1 Pratali RR, Hennemann SA, Amaral R, et al. Standardized terminology of adult spine deformity for brazilian portuguese. Coluna/ Columna 2015;14(04):281-285

2 Glassman SD, Bridwell K, Dimar JR, Horton W, Berven S, Schwab F. The impact of positive sagittal balance in adult spinal deformity. Spine (Phila Pa 1976) 2005;30(18):2024-2029

3 Lafage V, Schwab F, Patel A, Hawkinson N, Farcy JP. Pelvic tilt and truncal inclination: two key radiographic parameters in the setting of adults with spinal deformity. Spine (Phila Pa 1976) 2009;34(17):E599-E606

4 Schwab FJ, Blondel B, Bess S, et al; International Spine Study Group (ISSG) Radiographical spinopelvic parameters and disability in the setting of adult spinal deformity: a prospective multicenter analysis. Spine (Phila Pa 1976) 2013;38(13):E803-E812

5 Schwab F, Ungar B, Blondel B, et al. SRS-Schwab Adult Spinal Deformity Classification: AValidation Study. Spine (Phila Pa 1976) 2012;37(12):1077-1082

6 Terran J, Schwab F, Shaffrey CI, et al; International Spine Study Group. The SRS-Schwab adult spinal deformity classification: assessment and clinical correlations based on a prospective operative and nonoperative cohort. Neurosurgery 2013;73(04): 559-568

7 Schwab F, Dubey A, Gamez L, et al. Adult scoliosis: prevalence, SF36 , and nutritional parameters in an elderly volunteer population. Spine (Phila Pa 1976) 2005;30(09):1082-1085

8 Barreto MVA, Pratali RR, Barsotti CEG, Santos FPE, Oliveira CEAS, Nogueira MP. Incidence of spinal deformity in adults and its distribution according SRS-Schwab classification. Coluna/ Columna 2015;(14):93-96

9 Natarajan RN, Andersson GBJ. Lumbar disc degeneration is an equally important risk factor as lumbar fusion for causing adjacent segment disc disease. J Orthop Res 2017;35(01):123-130

10 Fu KMG, Rhagavan P, Shaffrey CI, Chernavvsky DR, Smith JS. Prevalence, severity, and impact of foraminal and canal stenosis among adults with degenerative scoliosis. Neurosurgery 2011;69 (06):1181-1187

11 Horton WC, Brown CW, Bridwell KH, Glassman SD, Suk SI, Cha $\mathrm{CW}$. Is there an optimal patient stance for obtaining a lateral 36 " radiograph? A critical comparison of three techniques. Spine (Phila Pa 1976) 2005;30(04):427-433

12 Vasconcelos IT, Sant'Anna DA, Oliveira CEAS, Herrero CFPS, Pratali RR. Escala de graduação da doença degenerativa lombar em exames de radiografia panorâmica da coluna vertebral. Coluna/ Columna 2019;18(01):32-36

13 Lafage R, Ferrero E, Henry JK, et al. Validation of a new computerassisted tool to measure spino-pelvic parameters. Spine J 2015;15 (12):2493-2502

14 Smith JS, Fu KMG, Urban P, Shaffrey CI. Neurological symptoms and deficits in adults with scoliosis who present to a surgical clinic: incidence and association with the choice of operative versus nonoperative management. J Neurosurg Spine 2008;9(04): 326-331

15 Pratali RR, Nasreddine MA, Diebo B, Oliveira CEAS, Lafage V. Normal values for sagittal spinal alignment: a study of Brazilian subjects. Clinics (São Paulo) 2018;73:e647

16 Lafage R, Schwab F, Challier V, et al;International Spine Study Group. Defining Spino-Pelvic Alignment Thresholds: Should Operative Goals in Adult Spinal Deformity Surgery Account for Age? Spine (Phila Pa 1976) 2016;41(01):62-68 